\title{
Deutsche Präpositionen als Träger lexikalischer Informationen. Eine Segmentierung nach Kriterien lexikalischer Autonomie
}

German prepositions as carriers of lexical information. A segmentation based on criteria of lexical autonomy

Rafael LÓPEZ-CAMPOS BODINEAU ${ }^{1}$

Universität Sevilla (Spain) / Universität Ateneum Gdansk

\begin{abstract}
This article is related to the study of the semantic load of the lexical category of prepositions. German prepositions, as in most Anglo-Germanic languages, are especially productive in the framework of two very specific syntactic functions: circumstantial complement and prepositional complement. Traditionally, in the context of the first one some type of semantic load is conferred on them, especially in cases where opposition pairs are available, while in the context of the second function they usually talk about uses of a purely conventional type with little lexical motivation. Through this article the possibility of relativizing this approach will be considered, and, thus, highlighting cases of prepositions with hardly any lexical load within the framework of a function as circumstantial complement, and, in the same way cases of prepositions with some kind of semantic value in the framework of the function as a repositional complement.
\end{abstract}

Keywords: Prepositions, Syntax, Syntactic Functions, Adverbial, Prepositional Object

1 (D) https://orcid.org/0000-0001-8482-4469. 


\section{Zusammenfassung}

Der vorliegende Artikel untersucht die Aufladung mit semantischer Bedeutung innerhalb der lexikalischen Kategorie der Präpositionen. Präpositionen in der deutschen Sprache sind, wie in den meisten anglo-germanischen Sprachen, im Rahmen zweier sehr bestimmter Satzglieder, der adverbialen Bestimmung und dem Präpositionalobjekt, besonders produktiv. Traditionell sind diese im ersten Fall, insbesondere dann, wenn es gegensätzliche Bedeutungspaare gibt, mit einer semantischen Bedeutung aufgeladen, während sie im Rahmen des zweiten Satzgliedes normalerweise eher rein konventionell und mit spärlicher lexikalischer Motivation verwendet werden. In diesem Artikel wird eine mögliche Relativierung dieses Ansatzes geprüft werden und so die Fälle der Präpositionen mit kaum lexikalischer Bedeutung im Rahmen des Satzglieds der adverbialen Bestimmung und, gleichermaßen, die Fälle von Präpositionen mit semantischem Wert im Rahmen des Satzglieds der Präpositionalobjekte hervorgehoben.

Schlüsselwörter: Präpositionen, Syntax, Syntaktische Funktionen, Adverbiale Bestimmungen, Präpositionalobjekt

\section{Problemstellung}

Der Gebrauch der Präpositionen ${ }^{2}$ - im Deutschen, aber auch in anderen Sprachen - artikuliert sich in und um Satzteile unterschiedlichster Beschaffenheit, wobei ich an dieser Stelle deren besondere Anwendung als einleitendes und kennzeichnendes Glied derjenigen Satzteile hervorheben möchte, die traditionell als adverbiale Bestimmungen (adverbiale Bestimmungen des Ortes, der Zeit, der Art und Weise, etc.) bezeichnet werden - dabei handelt es sich um ein Satzglied, das in der deutschen Sprache mit einer großen onomasiologischen Heterogenität ausgestattet ist: Umstandsbestimmung, Zirkumstanten, (freie Angaben), etc. - und deren Verwendung als Präpositionalobjekte 3 . Erstere stellen sich uns normalerweise

\footnotetext{
2 Beim vorliegenden Artikel handelt es sich um die nach der entsprechenden inhaltlichen und bibliografischen Prüfung erstellte deutsche Übersetzung und Überarbeitung des 2011 in der Zeitschrift Futhark (Sevilla) veröffentlichten Arbeit mit dem Titel „Las preposiciones alemanas como portadoras de información léxica. Una segmentación según criterios de autonimía léxica“.

3 Bekanntermaßen verfügen nicht alle Sprachen über eine Präpositionalsystem als zweckdienliches grammatikalisches Element für die Beschreibung der Beziehung zwischen einzelnen Gruppen, da diese in vielen durch Nominalflexion (Deklination) erfolgt. Die Verwendung dieser beiden Mechanismen, dem Präpositionalsystem und der Nominalflexion, ist dementsprechend antiproportional. Je größer die präpositionale Rekursivität ist, desto geringer ist die flektierende Rekursivität (siehe zum Beispiel die spanische Sprache mit einem starken Präpositionalsystem und folgerichtig keiner oder geringer Nominalflexion) und je größer die flektierende Rekursivität, desto geringer die präpositionale Rekursivität (siehe zum Beispiel die finnische Sprache mit starker nomineller Flexion und der daraus resultierenden eingeschränkten Verwendung von Präpositionen), ganz zu schweigen von intermediären
} 
als Elemente dar, denen eine Bedeutung innewohnt (bedeutungstragende Präpositionen), indem sie den existierenden semantisch-kognitiven Inhalt zwischen den Bedeutungen der nominellen Basen, die deren relationalen Wert untermauern, festlegen (mit Blick auf Minimalpaare, siehe den Wert von vor in das Auto steht vor dem Haus, im Gegensatz zu dem von hinter in das Auto steht hinter dem Haus). Zweitere sind Elemente ohne Bedeutung (nicht bedeutungstragende Präpositionen), da in diesem Fall die Verwendung der einen oder anderen weniger von deren semantischer Bedeutung - einer lange vorab etablierten semantischen Bedeutung - abhängt, als vielmehr von deren verbaler Basis, die diese hinsichtlich verpflichtender oder exemplarischer Regeln bedingt (siehe die Multifunktionalität der Basis vorbereiten in deren Vereinbarkeit mit für und auf in er bereitet sich auf die Reise vor und er bereitet sich für die Reise vor, ohne dass die beiden im Geringsten den Wert der bezeichneten Aktion bedingten. Gleichermaßen ist dies in der Verwendung zweier unterschiedlicher Präpositionen als Bestimmung einer gleichen Handlung vorzufinden, wie zum Beispiel im Falle von auf und an in der Kombination mit den Synonymen sich besinnen und sich erinnern, respektive sich auf etwas besinnen und sich an etwas erinnern, ohne die Möglichkeit an der präpositionalen Basis etwas zu verändern, wie bei ${ }^{*}$ sich an etwas besinnen und *sich auf etwas erinnern, oder sogar in der klar lokalisierbaren Konkurrenz zwischen einer Präpositionalphrase und einem Satzglied, wie zum Beispiel dem Genitivobjekt, wie sie in der Paarung sich an etwas erinnern und sich einer Sache erinnern auftaucht).

Stimmt es zwar, dass als Indikator für adverbiale Bestimmungen eingesetzte Präpositionen sich uns mit ihnen innewohnender Bedeutung, beziehungsweise der Tendenz dazu, präsentieren und dass als Indikator für Präpositionalobjekte eingesetzte Präpositionen keinerlei Bedeutung innewohnt, beziehungsweise diese dazu tendieren, so ist es - und dies ist unsere Ausgangs-hypothese - dennoch gewiss, dass dies in beiden Fällen nicht immer in semantisch homogener Weise auftritt. Mit anderen Worten haben weder alle Indikatoren von adverbialen Bestimmungen den gleichen Grad an lexikalischer Bedeutung (es gibt einige Fälle, in denen diese sogar bedeutungslos werden), noch weisen alle Indikatoren von Präpositionalobjekten das gleiche Maß an lexikalischer Bedeutungslosigkeit auf (in einigen Fällen präsentieren sich diese uns sogar voller semantischer

Zusammensetzungen, wie sie in germanischen Sprachen, durch eine Tendenz zur stärkeren Verwendung von Präpositionen, oder in slawischen Sprachen mit einer Tendenz zur Flexion, auftreten. In beiden Fällen bedeutet diese Tendenz aber keine Exklusivität. 
Signifikanz). Das Ziel dieses Artikels ist es daher, 1) zu analysieren, inwieweit es möglich ist, eine semasiologische Abstufung der Präpositionen innerhalb dieser beiden festgelegten Umgebungen vorzunehmen und 2) die Stadien aufzuzählen, in welche beide Umgebungen segmentiert werden können, wobei einzig die Bedeutung, die diese in dem einem oder anderen Fall in sich tragen, behandelt wird.

Unsere Arbeitsweise beinhaltet dabei die Analyse des Problems, indem wir die mögliche Kasuistik innerhalb jeder der beiden genannten Umgebungen, einerseits das Satzglied der adverbialen Bestimmung und andererseits das des Präpositionalobjekts, unter Abgrenzung eines Korpus und der Lokalisierung möglicher Bedeutungsebenen studieren. Letzteres geschieht mittels der Anwendungen dreier identifizierender semasiologischer Tests, nämlich 1) der größeren oder kleineren Möglichkeit ein minimales Bedeutungspaar festzustellen, insbesondere solche unterscheidenden Paare antagonistischen Charakters, bei welchen die Determinierung des unterscheidenden Charakters selbst ein Hinweis auf Bedeutung ist, 2) die größere oder kleinere Möglichkeit hierarchische Beziehungen herzustellen, indem die Artikulierung jedweder lexikalischen Umgebung in Felder und die dementsprechende Bedeutung, die deren Bestandteilen innewohnt, ein klares referentielles Bedeutungsmerkmal darstellt (vgl. Funktional-Lexematisches Modell nach Calañas Continente 2002, S. 25) und 3) die größere oder kleinere Möglichkeit der massenhaften Anwendung einer selben Präposition im Rahmen eines festgelegten Wertes.

\section{Präpositionen als Indikatoren adverbialer Bestimmungen}

Das Satzglied der adverbialen Bestimmung berücksichtigt, mit Hilfe von Deskriptoren unterschiedlichster Art, den Kontext oder das Umfeld in welchem die verbale Handlung stattfindet. Die gängigsten darunter gründen sich auf räumliche, zeitliche, modale, kausale und andere Dimensionen. Wenn wir in diesem Sinne den innerhalb des artikulierbaren Körpers der Präpositionen als Indikator des genannten Satzglieds verfügbaren Korpus betrachten, ist es derjenige, der mit der räumlichen Dimension assoziiert ist, der ohne jeden Zweifel ${ }^{4}$ die größte Rekursivität aufweist und aus welchem

${ }^{4}$ Von all jenen ist die räumliche Anwendung die vorherrschende, da sie die Grundlage bildet, auf die Bedeutung der anderen beruht. So ist zum Beispiel die temporale oder kausale Vorrangigkeit, auf die vor in Viertel vor acht oder er zittert vor Angst anspielt, respektive nicht mehr als die Projektion einer prototypisch räumlichen (vor als Signalwort einer räumlichen Voranstellung) kognitiven Grundlage auf andere Umgebungen. Aus diesem Grund werden wir unsere Analyse auf den räumlichen Korpus konzentrieren, während wir andere Anwendungen (temporal, modal, 
Elemente größter Produktivität wie an (seitliche Positionierung oder Verschiebung), auf (obere Positionierung oder Verschiebung), aus (äußere Positionierung oder Ver-schiebung), hinter (hintere Positionierung oder Verschiebung), in (innere Positio-nierung oder Verschiebung), unter (untere Positionierung oder Verschiebung), vor (vordere Positionierung oder Verschiebung), über (überräumliche Positionierung oder Verschiebung), zwischen (Positionierung zwischen zweien), unter (Po-sitionierung oder Verschiebung zwischen dreien oder mehr), $z u$ (typische richtungsweisende Präposition), bei (typische statische Präposition), gegenüber (frontale Positionierung), etc. besonders hervorstechen.

Mit dem Fokus auf der adverbialen Bestimmung und in Relation zu dem mit der räumlichen Dimension verknüpften Muster, sind wir der Ansicht, dass deren funktionelle Handhabung entsprechend dreier Kategorien erfolgen muss, die wir mit 1) nicht-lexikalisierte präpositionale Verwendung, 2) halblexikalisierte präpositionale Verwendung und 3) lexikalisierte präpositionale Verwendung, nach dem Ansatz von López-Campos Bodineau (1997), benennen.

Im Fall von nicht-lexikalisierten oder nicht-standardisierten Verwendungen müssen wir all jene präpositionalen Verwendungen zitieren, in deren Rahmen die Präposition ihre eigens ausgesprochene beschreibende Raum-Zeit-Dimension bewahrt. Es handelt sich um "motivierte" Verwendungen auf semantischer Ebene (Ullmann 1970, S. 104). Siehe folgende Beispiele:

\section{Der Stuhl steht vor dem Tisch Der Stuhl steht hinter dem Tisch Der Stuhl steht unter dem Tisch Der Stuhl steht auf dem Tisch (...)}

In all diesen Fällen nimmt die Präposition einen dominierenden Platz innerhalb des korrespondierenden semantischen Bereichs ein, in dem sie sich bewegt, während sie als unbestreitbarer Bedeutungsträger im Kontext der verbalen Handlung, in dem sie angewendet wird, auftritt. Die Assoziation dieser Verwendungen mit der tatsächlichen semantischen Bedeutung wird in diesen Fällen durch den Erfolg der Ausarbeitung der oben erwähnten Tests

kausal, etc.) außer Acht lassen, wodurch, da alle aus ersterem resultieren, ein kleinerer Korpus, sowohl in Bezug auf dessen Anwendungen und Kasuistik als auch folgerichtig auf dessen Häufigkeit, gebildet wird. 
unterstützt, nämlich 1) der Möglichkeit, minimale Bedeutungspaare antagonistischen Charakters zu bilden, die besonders bei Gegenüberstellungen der Typen auf / unter, vor / hinter usw. auffallen und ihre daraus resultierenden referentiellen Antagonismen, 2) der Möglichkeit, massenhafte Anwendungen der gleichen Präposition im Rahmen desselben Wertes vorzunehmen, wie wir an beliebigen zufälligen Ausdrücken wie an der Wand > an der Tür, an der Tafel etc.; auf dem Tisch > auf dem Stuhl, auf dem Boden etc. sehen können (Balzer, Díaz Alonso 2016: 14) und 3) der Möglichkeit, Beziehungen der verbalen Hierarchie innerhalb ihrer Umgebung aufzustellen, wie wir anhand der Beziehungen der verbalen Hyperonymie und Hyponymie bei der Verwendung einer jeden der beiden feststellen können. Siehe folgende Beispiele: etwas auf AKK legen, stellen, setzen, usw. (Hyperonyme) > etwas auf AKK schreiben, drucken, heften, kleben, usw. (Hyponyme).

Was die lexikalisierten Verwendungen betrifft, so handelt es sich um präpositionale Anwendungen, in deren Umfeld der Präposition eine semantische Bedeutungsaufladung fehlt, sodass ihre Anwendung einzig und allein aus der tatsächlichen Konvention resultiert. Wir sprechen insofern von "nicht-motivierten" Verwendungen, da uns die Verwendung dieser Präpositionen durch linguistische Traditionen vorgegeben ist, ohne dass dabei die Tatsache beeinträchtigt wird, dass wir in all diesen Fällen eine ursprüngliche Motivation erahnen können, die, einmal erzeugt, die Anwendung einer bestimmten Präposition archaisiert. Beispiele dieses Typs sind auf räumlicher Ebene gleichermaßen häufig, wie aus der folgenden Sammlung von Ausdrücken wie zu Hause in zu Hause sein, nach Hause in nach Hause gehen, zu Tisch in sich zu Tisch setzen, zu Bett in zu Bett gehen, etc. hervorgeht. Keine von ihnen bedient die zwei Test für Präpositionen mit nichtlexikalisiertem Charakter von den drei vorher zusammengestellten Tests. Das heißt, 1) in keinem Fall ist es möglich minimale Bedeutungspaare antagonistischen Charakters festzulegen, da diese normalerweise keine präpositionale Verwendung haben, die mit einem Wert ausgestattet wäre, der demjenigen gegenübergestellt ist, den uns irgendeiner der als Beispiel verwendeten Ausdrücke aufzeigt. Sollte er diesen doch haben, bewegt sich besagter Wert nicht im gleichen Lexikalisierungsbereich (siehe das lexikalisierte nach Hause gegenüber dem nicht-lexikalisierten von zu Hause) und 2) ist es in keinem Fall möglich eine massenhafte Anwendung irgendeines der Werte vorzunehmen, die keine der Präpositionen in sich tragen, wie wir in jedem der Beispiele zu Hause sein $>{ }^{*} z u$ Zimmer sein, ${ }^{*} z u$ Garten sein, etc. erkennen können. Sie erfüllen aber durchaus die dritte festgelegte Eigenschaft. So erlauben nämlich viele von ihnen durchaus hierarchische Beziehungen 
zwischen den ihre Verwendung einleitenden Verben, wie es in Korrelationen wie sich nach Hause hinbegeben, nach Hause laufen und nach Hause hinken zu erkennen ist, woraus wir eine klare Beziehung der Hyperonymie in sich nach Hause hinbegeben in Bezug auf nach Hause laufen, oder zu guter Letzt in Bezug auf nach Hause hinken wahrnehmen.

Als Zwischenkategorie machen wir in den Fällen eine große Gruppe halb-lexikalisierter Verwendungen ausfindig, in denen einerseits die Präposition eine Art Verbindung mit ihrer prototypischen kognitiven Dimension beibehält und andererseits ihre Anwendungen durch die Konvention gegeben sind (jene Konvention, die sich auf die semantischen Merkmale des Substantivs bezieht, das die Kernfunktion des von ihm eingeführten Syntagmas ausübt oder jene Konvention, die sich auf die traditionelle Verwendung bezieht, wie wir sie im Fall der lexikalisierten Verwendungen gesehen haben - zusammenfassend schlichtweg die Konvention $)^{5}$. In Bezug auf diese halb-lexikalisierten Verwendungen ist der folgende Zusammenhang zu beachten:

\begin{tabular}{|c|c|c|c|}
\hline Präp. & Nomineller Korpus & Positionierung & Direktionalität \\
\hline An & $\begin{array}{c}\text { Gewässer } \\
\text { (Küste, Strand, Fluss, } \\
\text { Meer, etc.) }\end{array}$ & Am Strand sein & An den Strand fahren \\
\hline An & $\begin{array}{c}\text { Studienzentren, wie zum } \\
\text { Beispiel Institutionen } \\
\text { (Universität, } \\
\text { Hochschule, etc.) }\end{array}$ & $\begin{array}{c}\text { An der Universität } \\
\text { studieren }\end{array}$ & An die Universität gehen \\
\hline
\end{tabular}

\footnotetext{
${ }^{5}$ Betrachten wir den Unterschied zwischen dieser Verwendungsart und der nicht-lexikalisierten anhand des Paares Er ist auf dem Tisch - Er ist auf dem Ausflug. Der erste Fall entspricht, wie bereits erläutert, einer prototypisch nicht-lexikalisierten Verwendung, da die Präposition ihre gesamte prototypische begrifflich-kognitive Bedeutung beibehält (er ist auf dem Tisch, gegenüber er ist unter dem Tisch, er ist an dem Tisch, er ist vor dem Tisch, er ist hinter dem Tisch, etc.). Im zweiten Fall jedoch ist die Verknüpfung von auf in Bezug auf die Basis Ausflug nicht so sehr durch die semantische Bedeutung der Präposition auf als vielmehr der nominellen Basis Ausflug gegeben, insofern als dass wir bei Anspielungen auf zeitlich ausdehnbare Aktivitäten eine automatische Anwendung dieser Präposition vermuten. Siehe auch andere Beispiele wie er ist auf dem Ausflug, er ist auf der Tagung, er ist auf dem Kongress, er ist auf der Reise, er ist auf der Hochzeit, etc. gegenüber inkorrekten Fällen wie *er ist in dem Ausflug, *er ist in der Tagung, ${ }^{*}$ er ist in dem Kongress, ${ }^{*}$ er ist in der Reise, ${ }^{*}$ er ist in der Hochzeit, etc.
} 


\begin{tabular}{|c|c|c|c|}
\hline Präp. & Nomineller Korpus & Positionierung & Direktionalität \\
\hline Auf & $\begin{array}{c}\text { Weite und/oder flache } \\
\text { und/oder gepflasterte } \\
\text { Fläche } \\
\text { (Straße, Hof, Platz, } \\
\text { Wiese, Terrasse, Balkon, } \\
\text { Bahnsteig, Gleis) }\end{array}$ & Auf der Straße sein & Auf die Straße gehen \\
\hline Auf & $\begin{array}{c}\text { Zeitlich ausdehnbare } \\
\text { Aktivitäten } \\
\text { (Hochzeit, Ausflug, } \\
\text { Kongress, etc.) }\end{array}$ & Auf einer Hochzeit sein & Auf eine Hochzeit gehen \\
\hline Auf & $\begin{array}{l}\text { Höhergelegene Orte } \\
\text { (Turm, Berg, etc.) }\end{array}$ & Auf dem Eiffelturm sein & $\begin{array}{c}\text { Auf den Eiffelturm } \\
\text { gehen }\end{array}$ \\
\hline Auf & $\begin{array}{l}\text { Offizielle öffentliche } \\
\text { Orte }\end{array}$ & $\begin{array}{c}\text { Auf der Post, auf der } \\
\text { Bank, auf dem Rathaus }\end{array}$ & $\begin{array}{l}\text { Auf die Post, auf die } \\
\text { Bank, aufs Rathaus }\end{array}$ \\
\hline Auf & $\begin{array}{c}\text { Benennung von Inseln } \\
\text { mit Artikel } \\
\end{array}$ & $\begin{array}{l}\text { Auf den Philippinen, auf } \\
\text { den kanarischen Inseln }\end{array}$ & $\begin{array}{c}\text { Auf die Philippinen, auf } \\
\text { die kanarischen Inseln }\end{array}$ \\
\hline In & $\begin{array}{c}\text { Örtlichkeiten } \\
\text { (Dorf, Land, Stadt) } \\
\end{array}$ & In der Stadt sein & In die Stadt fahren \\
\hline In & $\begin{array}{c}\text { Naturgebiete (Park, } \\
\text { Garten, Wald) }\end{array}$ & In einem Park sein & In einen Park gehen \\
\hline $\begin{array}{r}\text { In / } \\
\text { nach }\end{array}$ & Toponymik & $\begin{array}{c}\text { In New York } \\
\text { sein }\end{array}$ & $\begin{array}{c}\text { Nach New York } \\
\text { fliegen }\end{array}$ \\
\hline $\begin{array}{l}-/ \\
\text { Nach }\end{array}$ & $\begin{array}{c}\text { Adverbien (oben, unten, } \\
\text { vorne, hinten) }\end{array}$ & Oben sein & Nach oben gehen \\
\hline $\begin{array}{l}\text { In / } \\
\text { Nach }\end{array}$ & Himmelsrichtungen & Im Süden sein & Nach Süden gehen \\
\hline $\begin{array}{c}\mathrm{Zu} / \\
\mathrm{Bei}\end{array}$ & Physische Personen & $\begin{array}{l}\text { Bei meinen Eltern } \\
\text { wohnen }\end{array}$ & $\mathrm{Zu}$ meinen Eltern gehen \\
\hline $\mathrm{Zu}$ & Ziel, Zweck & - & $\begin{array}{c}\text { Zum Schwimmen gehen, } \\
\text { das Auto zur Reparatur } \\
\text { bringen, zum } \\
\text { Gottesdienst gehen, zum } \\
\text { Unterricht gehen }\end{array}$ \\
\hline
\end{tabular}

Im Falle dieser halb-lexikalisierten Verwendungen ist es 1) in keinem Fall möglich, minimale Bedeutungspaare mit antagonistischem Charakter zu bestimmen, da es normalerweise keine präpositionale Verwendung mit dem entgegengesetzten Wert zu den als Beispiele verwendeten Ausdrücken der Art in/aus, auf/unter, vor/hinter etc. gibt. Sollte es eine solche doch geben, bewegt sich dieser entgegengesetzte Wert nicht im gleichen Lexikalisierungsbereich 
(siehe das halb-lexikalisierte auf den Berg gegenüber dem nicht-lexikalisierten vom Berg). Dennoch ist es 2) möglich, eine massenhafte Anwendung eines der Werte, die jede dieser Präpositionen mit sich bringt, durchzuführen, wie aus einigen der soeben erwähnten Reihen ersichtlich ist (er ist auf dem Ausflug > er ist auf der Tagung, er ist auf dem Kongress, er ist auf der Reise, er ist auf der Hochzeit, etc.) und 3) ist es natürlich durchaus möglich, Hierarchiebeziehungen zwischen den einleitenden Verben dieser Verwendung herzustellen (sich auf einen Ausflug begeben > auf einen Ausflug gehen > auf einen Ausflug kriechen, etc.).

\section{Präpositionen als Kennzeichen von Präpositionalobjekten}

Bei den Präpositionalobjekten handelt es sich um Satzglieder auf Grundlage eines großen Korpus, die in sämtlichen Sprachen mit Internominalbeziehungen auf der Grundlage von Präpositionen zur Verfügung stehen. Die Präposition $a$ im spanischen Ausdruck renunciar a algo, die Präposition in im englischen Ausdruck to be interested in something oder die Präposition auf im deutschen Ausdruck auf etwas verzichten, etc. sind Exponenten eines Satzglieds, das auf der Anwendung eines Korpus beruht, der sich durch seine große syntaktische Hermetik auszeichnet, insofern als dass die Änderung einer dieser Präpositionen ungrammatische ${ }^{6}$ Zusammensetzungen erzeugen würde (Schierholz, 2001).

Im Falle der präpositionalen Verwendungen bei Satzgliedern der Präpositionalobjekte halten wir es im Unterschied $\mathrm{zu}$ adverbialen Bestimmungen für wichtig, einen Ausgangskorpus zusammenzustellen. Dies liegt daran, dass letztere leichter abgrenzbar sind als diejenigen, die im Rahmen der Präpositionalobjekte behandelt werden. Siehe, entnommen aus dem von Balzer, Barjau, Holst (1990) zusammengestellten Korpus:

An DAT zweifeln

Jemanden / etwas an DAT erkennen

Sich in AKK verlieben

Sich in DAT irren

Auf AKK verzichten

Sich auf AKK verlassen
Sich bei DAT um AKK bewerben

Sich bei DAT über AKK beklagen

Sich bei DAT über AKK beschweren

Sich bei DAT nach AKK erkundigen

Sich für AKK interessieren

Für AKK sorgen

\footnotetext{
${ }^{6}$ Siehe die Unmöglichkeit eines Einsetzens einer beliebigen anderen Präposition als $a$ in den spanischen Ausdruck renunciar a algo, einer anderen als in in den englischen Ausdruck to be interested in something oder einer anderen als auf in den deutschen Ausdruck auf etwas verzichten.
} 
Jemanden für AKK halten

Unter DAT leiden

$\mathrm{Zu} \mathrm{DAT} \mathrm{gehören}$

Jemandem für AKK danken

Über AKK herrschen

Sich an AKK erinnern

An AKK denken

An AKK glauben

An AKK etwas schreiben

Sich an AKK gewöhnen

Sich an AKK anpassen

Sich an DAT beteiligen

An DAT teilnehmen

An DAT teilhaben

An DAT Anteil haben

An DAT arbeiten

An DAT schreiben

An DAT bauen

An DAT komponieren

An DAT leiden

An DAT sterben

An DAT erkranken

Sich an DAT für AKK rächen

Sich an DAT für AKK auslassen

Auf AKK achten

Auf AKK aufpassen

Auf AKK aufmerksam sein

Auf AKK hinweisen

Auf AKK verweisen

Auf AKK hindeuten

Sich auf AKK beziehen

Sich aus AKK berufen

Etwas auf AKK beschränken

Etwas auf AKK konzentrieren

Etwas auf AKK begrenzen

Auf AKK verzichten

Sich auf AKK verlassen

Auf AKK zählen

Auf AKK vertrauen

Etwas auf AKK einstellen

Etwas auf AKK umstellen

Auf AKK hoffen
Sich bei jemandem für AKK entschuldigen

Sich an DAT für AKK rächen

Sich an DAT für AKK auslassen

Jemandem für AKK danken

Sich bei jemandem für AKK bedanken

Für AKK kämpfen

Für AKK sterben

Für AKK bürgen

Jemandem für AKK garantieren

Mit DAT diskutieren

Sich mit DAT streiten

Sich mit DAT unterhalten

Mit DAT kämpfen

Mit DAT ringen

Sich mit DAT vertragen

Sich mit DAT verloben

Sich mit DAT verheiraten

Sich mit DAT versöhnen

Mit DAT rechnen

Nach DAT forschen

Sich bei jemandem nach DAT erkundigen

Nach DAT fragen

Sich nach DAT sehnen

Sich bei DAT über AKK beschweren

Sich bei DAT über AKK beklagen

Über AKK diskutieren

Sich über AKK streiten

Über AKK kämpfen

Über AKK ringen

Über AKK schreiben

Über AKK sprechen

Über AKK nachdenken

Sich mit jemandem über AKK unterhalten

Sich über AKK freuen

Sich über AKK entrüsten

Über AKK staunen

Sich über AKK ärgern

Sich über AKK wundern

Über AKK weinen

Über AKK lachen

Sich um AKK bemühen

Sich um AKK bewerben 
Sich auf AKK freuen

Auf AKK warten

Sich auf AKK vorbereiten

Sich bei DAT für AKK bedanken

Sich bei DAT für AKK entschuldigen

Gegen AKK kämpfen

Sich mit DAT beschäftigen

Sich mit DAT befassen

Sich mit DAT konfrontieren

Sich mit DAT auseinandersetzen

Mit DAT anfangen

Mit DAT beginnen

Mit DAT enden

Mit DAT abschließen

Mit DAT sprechen
Um AKK kämpfen

Um AKK ringen

Sich um AKK streiten

Jmdn. um AKK bitten

Sich um AKK sorgen

Um AKK weinen

Sich um AKK kümmern

Sich um AKK fürchten

Jemanden von DAT befreien

Von DAT abhängen

Sich vor DAT fürchten

Jemanden vor DAT schützen

Vor DAT fliehen

Jemanden vor DAT warnen

Um AKK trauern

Um AKK gehen

Sich um AKK handeln

Auch im Falle der Präpositionalobjekte erkennen wir die drei bereits behandelten Ebenen der Lexikalisierung, d. h. nicht-lexikalisierte Verwendungen, lexikalisierte Verwendungen und gemischte oder halb-lexikalisierte Verwendungen.

Bei nicht-lexikalisierten Formationen handelt es sich also um einen Korpus, der durchaus eine Bedeutung oder zumindest eine relative Bedeutung innehat, da das Vorhandensein der einen oder anderen Präposition eine Aufladung mit semantische Bedeutung in sich selbst bewirkt, wie an den Paarungen an etwas schreiben / über etwas schreiben, an etwas komponieren / über etwas komponieren, an etwas dichten / über etwas dichten etc. erkannt werden kann. Siehe andere Gruppen:

\begin{tabular}{|c|c|c|}
\hline Regelung & Kognitives Umfeld & Bestand \\
\hline AN + AKK & $\begin{array}{c}\text { Referenz zu einem } \\
\text { Adressaten }\end{array}$ & $\begin{array}{c}\text { An AKK etwas schreiben, } \\
\text { telegraphieren, mailen, faxen, etc. }\end{array}$ \\
\hline AN + DAT & $\begin{array}{c}\text { Ausführung } \\
\text { erschaffender Arbeit }\end{array}$ & $\begin{array}{c}\text { An DAT arbeiten, schreiben, bauen, } \\
\text { komponieren, etc. }\end{array}$ \\
\hline
\end{tabular}




\begin{tabular}{|c|c|c|}
\hline Regelung & Kognitives Umfeld & Bestand \\
\hline $\mathrm{BEI}+\mathrm{DAT}$ & $\begin{array}{l}\text { Referenz zu einer Art } \\
\text { Instanz oder } \\
\text { Gesprächspartner }\end{array}$ & $\begin{array}{l}\begin{array}{c}\text { Sich bei DAT für AKK bedanken, } \\
\text { entschuldigen, etc. }\end{array} \\
\text { Sich bei DAT um AKK bewerben, etc. } \\
\text { Sich bei DAT über AKK beklagen, } \\
\text { beschweren, etc. } \\
\text { Sich bei DAT nach AKK erkundigen, etc. }\end{array}$ \\
\hline FÜR + AKK & $\begin{array}{l}\text { Referenz zugunsten von } \\
\text { etwas oder jemandem }\end{array}$ & $\begin{array}{l}\text { Für AKK kämpfen, sterben, bürgen, } \\
\text { garantieren, etc. }\end{array}$ \\
\hline GEGEN + AKK & $\begin{array}{c}\text { Referenz gegen etwas } \\
\text { oder jemanden }\end{array}$ & Gegen AKK kämpfen, ringen, etc. \\
\hline $\mathrm{MIT}+\mathrm{DAT}$ & $\begin{array}{l}\text { Widmung einer } \\
\text { Angelegenheit }\end{array}$ & $\begin{array}{l}\text { Sich mit DAT beschäftigen, befassen, } \\
\text { konfrontieren, auseinandersetzen, etc. } \\
\text { Mit DAT anfangen, beginnen, enden, } \\
\text { abschließen, etc. }\end{array}$ \\
\hline $\mathrm{MIT}+\mathrm{DAT}$ & $\begin{array}{l}\text { Referenz zu einem } \\
\text { Gesprächspartner }\end{array}$ & $\begin{array}{l}\text { Mit DAT sprechen, diskutieren, etc. } \\
\text { Sich mit DAT streiten, unterhalten, etc. } \\
\text { Mit DAT kämpfen, ringen, etc. } \\
\text { Sich mit DAT vertragen, verloben, } \\
\text { verheiraten, versöhnen, etc. }\end{array}$ \\
\hline $\mathrm{NACH}+\mathrm{DAT}$ & Suche nach etwas & $\begin{array}{l}\text { Nach DAT forschen, fragen, etc. } \\
\begin{array}{l}\text { Sich bei jemandem nach DAT } \\
\text { erkundigen, etc. } \\
\text { Sich nach DAT sehnen, etc. }\end{array}\end{array}$ \\
\hline ÜBER + AKK & $\begin{array}{c}\text { Referenz zum Ursprung, } \\
\text { Motiv oder Grund von } \\
\text { etwas }\end{array}$ & $\begin{array}{l}\text { Sich bei DAT über AKK beschweren, } \\
\text { beklagen, etc. } \\
\text { Über AKK diskutieren, kämpfen, ringen, } \\
\text { etc. } \\
\text { Sich über AKK streiten, etc. }\end{array}$ \\
\hline
\end{tabular}




\begin{tabular}{|c|c|c|}
\hline Regelung & Kognitives Umfeld & Bestand \\
\hline ÜBER + AKK & $\begin{array}{l}\text { Darstellung einer } \\
\text { Thematik }\end{array}$ & $\begin{array}{l}\text { Über AKK schreiben, sprechen, } \\
\text { nachdenken, etc. } \\
\text { Sich mit jemandem über AKK } \\
\text { unterhalten, etc. }\end{array}$ \\
\hline ÜBER + AKK & $\begin{array}{l}\text { Darstellung eines } \\
\text { Gefühls }\end{array}$ & $\begin{array}{l}\text { Sich über AKK freuen, entrüsten, ärgern, } \\
\text { wundern, etc. } \\
\text { Über AKK staunen, weinen, lachen, etc. }\end{array}$ \\
\hline $\mathrm{UM}+\mathrm{AKK}$ & $\begin{array}{c}\text { Erfolgsorientierte } \\
\text { Handlung }\end{array}$ & $\begin{array}{l}\text { Sich um AKK bemühen, bewerben, } \\
\text { streiten, etc. } \\
\text { Um AKK kämpfen, ringen, etc. } \\
\text { Jemanden um AKK bitten, etc. }\end{array}$ \\
\hline $\mathrm{UM}+\mathrm{AKK}$ & $\begin{array}{l}\text { Ausdruck gewisser } \\
\text { Sorge um jemanden }\end{array}$ & $\begin{array}{l}\text { Sich um AKK sorgen, kümmern, } \\
\text { fürchten, etc. } \\
\text { Um AKK weinen, trauen, etc. }\end{array}$ \\
\hline
\end{tabular}

In all diesen Fällen ist es 1) nicht möglich, minimale Bedeutungspaare mit antagonistischem Charakter zu bestimmen, da der Wert der Präpositionen keinen Hinweis auf Werte erkennen lässt, die solche Antagonismen herstellen könnten. Es ist aber 2) durchaus möglich, eine massenhafte Anwendung eines beliebigen Wertes, der einer dieser Präpositionen innewohnt, durchzuführen, wie an Abfolgen wie an etwas schreiben > an etwas komponieren, an etwas dichten, etc. erkannt werden kann und es ist 3) auch möglich, innerhalb dieses Musters Hierarchiebeziehungen herzustellen (an etwas arbeiten > an etwas schreiben > an etwas dichten, oder über etwas arbeiten > über etwas schreiben > über etwas dichten). Siehe:

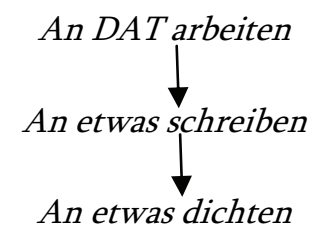

In Bezug auf diese dritte Bedingung und ebenso wie in den präpositionalen Verwendungen im Falle der adverbialen Bestimmungen festgelegt, können wir im Rahmen dieser Beziehungen die übliche proleptische 
Beziehung dieser Fälle bestimmen, die durch das Vorhandensein des Hyperonyms (an etwas arbeiten, im Fall des von uns erwähnten Beispiels) in der Darstellung der semantischen Basis jeder der Einheiten, die Gegenstand der Analyse sein könnten, gekennzeichnet ist: an etwas bauen < bauen, indem man an etwas arbeitet / an etwas arbeiten und dabei bauen, schreiben < schreiben, indem man an etwas arbeitet / an etwas arbeiten und dabei schreiben, an etwas komponieren < komponieren, indem man an etwas arbeitet / an etwas arbeiten und dabei komponieren, etc. ${ }^{7}$

Auf der anderen Seite finden wir auch im Rahmen des Präpositionalobjekts Verwendungen, die als lexikalisiert bezeichnet werden können, wie zum Beispiel jmdn. zu etwas einladen oder an etwas zweifeln, etc. in deren Fall eine präpositionale Verwendung vorliegt, die auf kein anderes Muster übertragen werden kann. Das bedeutet, der übergeordnete Wert von einladen in der Satzstruktur jmdn. zu etwas einladen ist dank der Applikation von $z u$ auf keine andere Konstruktion übertragbar, weswegen sich es um eine Verwendung isolierten Charakters handelt. Exakt das Gleiche tritt in den Fällen von an etwas zweifeln oder auf etwas verzichten und anderen auf. Mit anderen Worten haben wir in diesen Fällen weder eine Möglichkeit kohiponymische Beziehungen noch irgendeine Form der Hyperonymie oder Hyponymie festzustellen. Im Falle dieser Zusammensetzungen findet keines der drei Kriterien Anwendung, es ist also 1) nicht möglich ein kleinstes Bedeutungspaar mit antagonistischem Charakter festzustellen, da der Wert der Präpositionen keinen Hinweis auf solche Werte erkennen lässt, die solche Antagonismen herstellen könnten, 2) auch nicht möglich, da es sich um Applikationen isolierten Charakters handelt, eine massenhafte Anwendung irgendeines der Werte durchzuführen, die irgendeiner der Präpositionen innewohnen und folgerichtig ist es 3) nicht möglich hierarchische Beziehungen innerhalb des Beispiels herzustellen.

Und schließlich lässt sich das Bestehen einer Gruppe vermuten, die wir als halb-lexikalisiert bezeichnen könnten, gebildet durch Verben, deren prototypische Präpositionalobjekt mit einer Gebrauchskonstante verknüpfbar sind (siehe die Gruppe gebildet durch an einer Sache teilnehmen, an einer Sachen teilhaben, sich an einer Sache beteiligen und an einer Sache Anteil nehmen, etc.). Siehe einige weiter Gruppen:

\footnotetext{
7 Die Kategorien der Unveränderlichkeit und der lexikalischen Fülle sind daher entgegengesetzt proportional, da die Präposition insbesondere in den Fällen eine gewisse Bedeutung annimmt, in denen ein und dasselbe Verb mit unterschiedlichen Konnektoren verbunden wird.
} 


\begin{tabular}{|c|c|c|}
\hline Regelung & Kognitives Umfeld & Bestand \\
\hline $\mathrm{AN}+\mathrm{AKK}$ & Geistige Aktivität & $\begin{array}{c}\text { Sich an AKK erinnern } \\
\text { An AKK denken } \\
\text { An AKK glauben }\end{array}$ \\
\hline $\mathrm{AN}+\mathrm{AKK}$ & Anpassung an etwas & $\begin{array}{l}\text { Sich an AKK gewöhnen } \\
\text { Sich an AKK anpassen }\end{array}$ \\
\hline $\mathrm{AN}+\mathrm{DAT}$ & Teilnahme an etwas & $\begin{array}{l}\text { Sich an DAT beteiligen } \\
\text { An DAT teilnehmen } \\
\text { An DAT teilhaben } \\
\text { An DAT Anteil haben }\end{array}$ \\
\hline $\mathrm{AN}+\mathrm{DAT}$ & Verweis auf eine Krankheit & $\begin{array}{c}\text { An DAT leiden } \\
\text { An DAT sterben } \\
\text { An DAT erkranken }\end{array}$ \\
\hline $\mathrm{AN}+\mathrm{DAT}$ & Verweis auf Grausamkeiten & $\begin{array}{l}\text { Sich an DAT für AKK rächen } \\
\text { Sich an DAT für AKK auslassen }\end{array}$ \\
\hline $\mathrm{AUF}+\mathrm{AKK}$ & $\begin{array}{l}\text { Widmung oder } \\
\text { Aufmerksamkeit für etwas } \\
\text { oder jemanden }\end{array}$ & $\begin{array}{c}\text { Auf AKK achten } \\
\text { Auf AKK aufpassen } \\
\text { Auf AKK aufmerksam sein }\end{array}$ \\
\hline $\mathrm{AUF}+\mathrm{AKK}$ & $\begin{array}{l}\text { Feststellung einer Art } \\
\text { Referenz }\end{array}$ & $\begin{array}{c}\text { Auf AKK hinweisen } \\
\text { Auf AKK verweisen } \\
\text { Auf AKK hindeuten } \\
\text { Sich auf AKK beziehen } \\
\text { Sich aus AKK berufen } \\
\end{array}$ \\
\hline $\mathrm{AUF}+\mathrm{AKK}$ & $\begin{array}{l}\text { Physische oder psychische } \\
\text { Begrenzung }\end{array}$ & $\begin{array}{c}\text { Etwas auf AKK beschränken } \\
\text { Etwas auf AKK konzentrieren } \\
\text { Etwas auf AKK begrenzen } \\
\text { Auf AKK verzichten }\end{array}$ \\
\hline $\mathrm{AUF}+\mathrm{AKK}$ & Ausdruck von Vertrauen & $\begin{array}{c}\text { Sich auf AKK verlassen } \\
\text { Auf AKK rechnen } \\
\text { Auf AKK zählen } \\
\text { Auf AKK vertrauen } \\
\end{array}$ \\
\hline $\mathrm{AUF}+\mathrm{AKK}$ & Anpassung an etwas & $\begin{array}{l}\text { Etwas auf AKK einstellen } \\
\text { Etwas auf AKK umstellen }\end{array}$ \\
\hline $\mathrm{AUF}+\mathrm{AKK}$ & Ausdruck einer Erwartung & $\begin{array}{c}\text { Auf AKK hoffen } \\
\text { Sich auf AKK freuen } \\
\text { Auf AKK warten } \\
\text { Auf AKK rechnen } \\
\text { Sich auf AKK vorbereiten }\end{array}$ \\
\hline
\end{tabular}




\begin{tabular}{|c|c|c|}
\hline Regelung & Kognitives Umfeld & Bestand \\
\hline & & Sich vor DAT fürchten \\
VOR + DAT & Ausdruck einer Art Angst & Jemanden vor DAT schützen \\
& & Vor DAT fliehen \\
& & Jemanden vor DAT warnen \\
\hline
\end{tabular}

In all diesen Fällen ist es 1) nicht möglich kleinste Bedeutungspaare antagonistischen Charakters festzustellen, da der Wert der Präpositionen keinen Hinweis auf die Sorte Werte erkennen lässt, die solche Antagonismen herstellen könnten und 2) auch nicht möglich hierarchische Beziehungen innerhalb des Beispiels herzustellen. Es ist jedoch 3) möglich eine massenhafte Anwendung irgendeines der Werte durchzuführen, die irgendeiner der Präpositionen innewohnen, wie sie in Satzreihen wie an einer Sache teilnehmen > an einer Sache teilhaben > sich an einer Sache beteiligen > an einer Sache Anteil nehmen, etc. erkennbar sind.

\section{Schlussfolgerung}

Gegründet auf das in diesem Artikel Behandelte, können wir folgende Schlussfolgerungen ziehen:

1. Präpositionen sind nicht immer Träger der gleichen Art semantischer Bedeutung. In einigen Fällen ist diese semantische Aufladung klarer als in anderen. So sind die zwei Satzglieder, die adverbiale Bestimmung und das Präpositionalobjekt, von denen die Präpositionen prototypische Kennzeichen sind, wobei die mit ihnen zu verbindende lexikalische Relevanz der sie einführenden Präpositionen berücksichtigt wird, auf drei Ebenen artikulierbar. Es handelt sich dabei um lexikalisierte Verwendungen, halb-lexikalisierte Verwendungen und nicht-lexikalisierte Verwendungen. Der lexikalisierte Korpus ist durch präpositionale Verwendungen gegeben, die sich aus Archaisierungen ergeben, die keine Möglichkeit haben, das Kennzeichen mit einleitendem präpositionalem Charakter zu verändern (siehe den semantischen Unterschied zwischen dem Ausdruck zu Hause und den Ausdrücken im Hause oder in dem Hause sowie die formale Unbeweglichkeit des ersteren gegenüber dem letzteren). Der nicht-lexikalisierte Korpus ist durch präpositionale Verwendungen mit klarer semantischer Motivation gegeben, sofern - in diesem Fall schon - die entsprechenden Grundlagen aus Kontextgründen veränderbar sind (siehe er steht vor der Tür gegenüber er steht hinter der Tür oder er steht an der Tür, etc.). Schließlich wirkt der halb-lexikalisierte Korpus 
an den beiden vorhergehenden insofern mit, als dass eine durchaus transparente semantische Motivation wahrgenommen wird, ohne dass jedoch die Grundlagen kontextbedingt verändert werden können (siehe er ist auf dem Ausflug gegenüber er ist in dem Ausflug).

2. Aufgrund der mehr oder weniger lexikalisierten Dimension der Präposition, stellen wir eine mehr oder weniger starke Anwendung der drei vorab aufgestellten Tests fest, nämlich die größere oder kleinere Möglichkeit kleinste Bedeutungspaare zu bestimmen, vor allem jener antagonistischen Charakters, bei welchen die Determinierung des unterscheidenden Merkmals selbst ein Bedeutungskennzeichen ist, die größere oder kleinere Möglichkeit hierarchische Beziehungen zu bestimmen, insofern als dass die Artikulation jeder lexikalischen Umgebung in gewissen Feldern und die daraus folgende Abgrenzung der ihren einzelnen Einheiten innewohnenden Bedeutung, ein klares Referenzmarke der Bedeutung darstellt, sowie die größere oder kleinere Möglichkeit ein und dieselbe Präposition im Rahmen einer determinierten Bedeutung in massivem Umfang anzuwenden.

3. Bei den als lexikalisch festgelegten Verwendungen beobachten wir, dass sich im Falle der Präpositionen als Einleitung adverbialer Bestimmungen alle drei Tests anwenden lassen und immerhin zwei der Tests, werden die Präpositionen als Einleitung von Präpositionalobjekten verwendet. Auf der anderen Seite stellen wir bei den nicht-lexikalisierten Verwendungen fest, dass sich im Falle des Gebrauchs als Einleitung adverbialer Bestimmungen ein einziger Test anwenden lässt, im Falle des Gebrauchs als Einleitung von Präpositionalobjekten sogar kein einziger. Zu guter Letzt stellen wir fest, dass bei den von uns als halb-lexikalisiert definierten Verwendungen im Falle des Gebrauchs als Einleitung adverbialer Bestimmungen zwei der Tests anwendbar sind und im Falle des Gebrauchs als Einleitung von Präpositionalobjekten eine einzige.

\begin{tabular}{|c|c|c|c|}
\hline $\begin{array}{c}\text { Durch Präposition } \\
\text { gebildetes Satzglied }\end{array}$ & $\begin{array}{c}\text { Begrenzung } \\
\text { kleinster } \\
\text { Bedeutungspaare } \\
\text { antagonistischen } \\
\text { Charakters }\end{array}$ & $\begin{array}{c}\text { Umfangreiche } \\
\text { Anwendung des } \\
\text { Musters }\end{array}$ & $\begin{array}{c}\text { Begrenzung der } \\
\text { hierarchischen } \\
\text { Beziehungen } \\
\text { (Hyponymie / } \\
\text { Hyperonymie) }\end{array}$ \\
\hline $\begin{array}{l}\text { Adverbiale Bestimmung - } \\
\text { nicht-lexikalisiert }\end{array}$ & JA & JA & JA \\
\hline
\end{tabular}




\begin{tabular}{|l|c|c|c|}
\hline $\begin{array}{l}\text { Adverbiale Bestimmung - } \\
\text { lexikalisiert }\end{array}$ & NEIN & NEIN & JA \\
\hline $\begin{array}{l}\text { Adverbiale Bestimmung - } \\
\text { halb-lexikalisiert }\end{array}$ & NEIN & JA & JA \\
\hline \multicolumn{3}{|l|}{} \\
\hline $\begin{array}{l}\text { PRÄPOSITIONALOBJEKT } \\
\text { nicht-lexikalisiert }\end{array}$ & NEIN & JA & JA \\
\hline $\begin{array}{l}\text { PRÄPOSITIONALOBJEKT } \\
\text { lexikalisiert }\end{array}$ & NEIN & NEIN & NEIN \\
\hline $\begin{array}{l}\text { PRÄPOSITIONALOBJEKT } \\
\text { halb-lexikalisiert }\end{array}$ & NEIN & JA & NEIN \\
\hline
\end{tabular}

4. Entsprechend all dieser Feststellungen, zeigt die Verwendung von als Einleitung adverbialer Bestimmungen verwendeten Präpositionen eine größere Beständigkeit als diejenige, die sich auf die Präpositionalobjekte bezieht, da in ersterem Fall eine häufigere positive Resonanz auf die unterschiedlichen Tests erkennbar ist als in zweiterem. Siehe:

\begin{tabular}{|c|c|c|c|}
\hline $\begin{array}{c}\text { Präpositionale } \\
\text { Verwendung }\end{array}$ & $\begin{array}{c}\text { Anzahl positiver } \\
\text { Ergebnisse bei } \\
\text { nicht- } \\
\text { lexikalisierten } \\
\text { Fällen }\end{array}$ & $\begin{array}{c}\text { Anzahl positiver } \\
\text { Ergebnisse bei } \\
\text { halb-lexikalisierten } \\
\text { Fällen }\end{array}$ & $\begin{array}{c}\text { Anzahl positiver } \\
\text { Ergebnisse bei } \\
\text { lexikalisierten } \\
\text { Fällen }\end{array}$ \\
\hline $\begin{array}{c}\text { Adverbiale } \\
\text { Bestimmung }\end{array}$ & 3 & 2 & 1 \\
\hline Präpositionalobjekt & 2 & 1 & 0 \\
\hline
\end{tabular}

\section{Bibliografie}

Balzer, B., Barjau, E., Holst, K. (1990). La lengua alemana: su complemento preposicional. Barcelona: Ariel.

Balzer, B., Díaz Alonso, J. (2006). Die Präposition als Präverb im Deutschen. Madrid: Ediciones Universidad Autónoma.

Calañas Continente, J. A. (2002). El dominio léxico "Existencia" en alemán. Frankfurt: Peter Lang.

Gierden Vega, C., Heinsch, B. (2001). Strukturen - Manual práctico de la lengua y gramática alemanas. Valladolid: Secretariado de publicaciones Universidad de Valladolid. 
López-Campos Bodineau, R. (1997). Los preverbios separables en lengua alemana. Sevilla: Secretariado de Publicaciones Universidad de Sevilla.

López-Campos Bodineau, R. (2003). Morfosintaxis Alemana. Barcelona: Herder.

Müller, W. (2012). Wörterbuch deutscher Präpositionen. Die Verwendung als Anschlu $\beta$ an Verben, Substantive, Adjektive und Adverbien. Berlin: de Gruyter.

Schierholz, S. J. (2001). Präpositionalattribute. Tübingen: Niemeyer.

Schmitz, W. (1996). Der Gebrauch der deutschen Präpositionen. München: Hueber.

Ullmann, S. (1970). Semántica. Madrid: Aguilar.

Wullen, F. (2019). Der syntaktische Gebrauch der Präpositionen. Inktank Publishing. 
